\section{Escala de apoio social aplicada a uma população de gestantes: confiabilidade teste-reteste e estrutura de concordância dos itens}

\author{
Social support scale: test-retest reliability \\ in pregnant women and structures of agreement \\ and disagreement
}

Kátia Silveira da Silva 1

Evandro Silva Freire Coutinho ${ }^{2,3}$

\title{
Introdução
}

1 Instituto Fernandes

Figueira, Fundação Oswaldo Cruz, Rio de Janeiro, Brasil.

2 Escola Nacional de Saúde Pública, Fundação Oswaldo Cruz, Rio de Janeiro, Brasil.

3 Instituto de Medicina Social, Universidade do Estado do Rio de Janeiro, Rio de Janeiro, Brasil.

\section{Correspondência} K. S. Silva

Núcleo de Epidemiologia, Instituto Fernandes Figueira, Fundação Oswaldo Cruz. Rua Belisario Távora 129, apto. S101, Rio de Janeiro, $R J$ 22245-070, Brasil. katiass@iff.fiocruz.br

\begin{abstract}
There is a growing interest in research on the as-
Abstract sociation between social support and health outcomes. The objective of this study was to evaluate the reliability and structure of agreement of a social support scale. Test-retest reliability was measured in a group of pregnant women $(n=65)$ in a public maternity ward. Intraclass correlation coefficient (ICC) and quadractically weighted kappa $\left(k w^{2}\right)$ were used as agreement measures. Log-linear statistical models were fitted to describe patterns of agreement. ICC for social support score was 0.90. The kw2 ranged from 0.23 to 0.70. Log-linear models that provided the best fit to the data were diagonal agreement plus linear-by-linear association and quasi-independence models. The scale was considered a reliable instrument to measure social support scale in low-income pregnant women.
\end{abstract}

Social Support; Validation Studies; Linear Models
A investigação da relação existente entre apoio social e desfechos relacionados à saúde materna e perinatal tem despertado a atenção de pesquisadores nos últimos vinte anos.

$\mathrm{O}$ apoio social foi avaliado por intermédio da escala desenvolvida no "Medical Outcomes Study”, que mede a percepção dos indivíduos sobre o grau de apoio social 1. Este instrumento foi submetido a procedimentos padronizados de tradução e versão pelo Estudo Pró-Saúde 2 , tendo sido recomendada a sua utilização após análise de confiabilidade e validade ${ }^{3}$. Deste modo, o instrumento foi aplicado a um grupo de mulheres no período gravídico-puerperal participantes de um estudo com objetivo de avaliar o papel do apoio social como fator de proteção para pré-eclâmpsia.

Como a confiabilidade depende do conjunto do processo de aferição, justifica-se que o desempenho deste instrumento também seja avaliado no contexto do nosso estudo 4 . Além disso, deve-se considerar o caráter subjetivo do apoio social e os diferentes conceitos adotados, para os quais não se têm padrões de respostas. Estas características tornam relevante a análise da confiabilidade para avaliar a qualidade das informações que envolveram este constructo. 


\section{Material e métodos}

A população do estudo foi composta por uma amostra aleatória estratificada, segundo enfermaria, de 65 mulheres internadas durante a gestação ou parto (entre agosto e outubro de 2003) numa maternidade pública do Rio de Janeiro.

A escala de apoio social é constituída por 19 itens relacionados com cinco dimensões de apoio. Cada item busca medir numa escala ordinal a freqüência com que a gestante acredita dispor de apoio social.

As respostas foram obtidas por meio da realização de duas entrevistas pelo mesmo avaliador, com intervalos de até sete dias, após assinatura do Termo de Consentimento. A estabilidade da medida de apoio social foi avaliada pela confiabilidade teste-reteste do escore total da escala e de cada dimensão calculada pelo coeficiente de correlação intraclasse (CCIC) com dois fatores e efeitos fixos (two-way fixedeffects) 5 . A consistência interna dos itens de cada dimensão foi avaliada pelo $\alpha$-Cronbach 4 .

$\mathrm{Na}$ análise da confiabilidade teste-reteste dos itens utilizou-se o kappa com ponderação quadrática para estimar a concordância de cada item, definindo pesos diferenciados de acordo com o grau de discordância 6 .

Por fim, foram testados modelos log-lineares para descrever o padrão de concordância existente entre as respostas. Explicações sobre cada um desses modelos encontram-se em outros estudos sobre o tema 3,7 . Usou-se uma freqüência de 0,5 quando alguma célula da diagonal da tabela de contingência era zero. A medida de adequação do ajuste do modelo foi a estatística $\chi^{2}$ da razão de verossimilhança - resultante da comparação dos valores "preditos" pelos modelos e dos valores "observados", sob hipótese da independência. Considerou-se modelo de melhor ajuste aquele que combinasse dois critérios: o critério da parcimônia e do menor valor de $G^{2}$ para o grau de liberdade (g.l.) correspondente. No caso de modelos hierárquicos embutidos, baseou-se na comparação das estatísticas $G^{2}$, optando-se por uma modelagem mais complexa quando a inclusão de termos implicasse uma contribuição para o ajuste estatisticamente significativa 7 .

\section{Resultados}

A população de estudo foi constituída por mulheres com idade média de 27 anos ( $D P=7$ ), cuja escolaridade esteve distribuída entre o nível elementar (50\%) e o nível médio (50\%). As medianas dos escores totais da escala nos dois momentos foram 77 pontos na primeira e de 78 pontos na segunda entrevista.

O CCIC para o escore total foi de 0,90 (IC95\%: 0,84-0,94). A dimensão emocional apresentou o maior nível de confiabilidade $(\mathrm{CCIC}=0,85)$. Os valores do CCIC segundo a dimensão, assim como o $\alpha$ de Cronbach encontram-se na Tabela 1. Não foram constatadas diferenças estatisticamente significativas nos valores do CCIC entre grupos diferentes de idade, escolaridade, de idade gestacional e de entrevistadores.

Os kappas ponderados estimados para cada item variaram entre 0,23 e 0,70. O desempenho mais fraco se refere ao item 5 relacionado à dimensão afetiva (Tabela 2).

$\mathrm{O}$ modelo que melhor se ajustou ao maior número de itens foi o de semi-independência (1, $5,6,7,13,17,18$ ), seguido do modelo de concordância diagonal mais associação linear por linear $(2,3,10,19)$, e dos modelos de concordância diagonal $(4,12,14)$ de associação linear por linear $(8,9,11)$, parâmetros triangulares (16). Para o item 15 não foi possível identificar pelos modelos testados um padrão de concordância e discordância presente nos dados (Tabela 3).

\section{Discussão}

A versão da escala de apoio social mostrou, quando aplicada por intermédio de entrevista a uma população de gestantes de baixa escolaridade, um índice alto de confiabilidade semelhante ao descrito no Estudo Pró-Saúde, cuja população apresentava grau de escolaridade mais elevado ${ }^{3}$. A avaliação da confiabilidade teste-reteste do escore total revelou um nível excelente de concordância, que sofreu uma redução no momento em que se analisou esta propriedade de acordo com as dimensões. No caso da dimensão afetiva, por exemplo, esta redução pode ter sido causada por uma maior instabilidade das respostas ou ainda pelo fato de esta dimensão ser composta por apenas três itens, o que a tornaria mais sensível às variações entre os escores. O tamanho amostral deste estudo pode ter contribuído para uma menor precisão das estimativas destes indicadores, no entanto, os limites inferiores dos intervalos de confiança ainda apresentaram um grau de concordância acima de moderado em relação às dimensões e ao escore total, apesar de isoladamente alguns itens da escala terem tido um fraco desempenho.

A análise da consistência interna também mostrou índices adequados. Este coeficiente, quando é mais elevado que 0,90 , pode sugerir a 
Coeficiente de Correlação Intraclasse $(\mathrm{CCIC})$ e $\alpha$-Cronbach para dimensões da escala de apoio social aplicada a gestantes de uma maternidade pública. Rio de Janeiro, Brasil, 2003.

\begin{tabular}{lccc}
\hline Dimensões & CCIC (IC95\%) & $\alpha$-Cronbach Teste & $\alpha$-Cronbach Reteste \\
\hline Informação & $0,66(0,50-0,78)$ & 0,78 & 0,80 \\
Emocional & $0,85(0,76-0,90)$ & 0,74 & 0,75 \\
Material & $0,66(0,50-0,78)$ & 0,63 & 0,74 \\
Afetivo & $0,58(0,39-0,72)$ & 0,78 & 0,65 \\
Interação social & $0,74(0,60-0,83)$ & 0,73 & 0,80 \\
Escore total & $0,90(0,84-0,94)$ & 0,91 & 0,91 \\
\hline
\end{tabular}

Tabela 2

Confiabilidade teste-reteste (kappa com ponderação quadrática) da escala de apoio social aplicada a gestantes de uma maternidade pública. Rio de Janeiro, Brasil, 2003.

\begin{tabular}{|c|c|c|}
\hline Dimensões & Se você precisar, com que freqüência conta com alguém & Kappa ponderado (IC95\%) \\
\hline \multirow[t]{4}{*}{ Emocional } & 2. para lhe ouvir & $0,50(0,29-0,66)$ \\
\hline & 8. para confiar e falar dos seus problemas & $0,48(0,27-0,65)$ \\
\hline & 15. compartilhar preocupações e medos mais íntimos & $0,62(0,44-0,75)$ \\
\hline & 18. que compreenda seus problemas & $0,68(0,52-0,79)$ \\
\hline \multirow[t]{4}{*}{ Material } & 1. que o ajude se ficar de cama & $0,49(0,28-0,66)$ \\
\hline & 4. levá-lo ao médico & $0,52(0,32-0,68)$ \\
\hline & 11. preparar refeições & $0,70(0,56-0,81)$ \\
\hline & 14. tarefas diárias se ficar doente & $0,48(0,27-0,65)$ \\
\hline \multirow[t]{4}{*}{ Informação } & 3.para lhe dar bom conselho numa situação de crise & $0,56(0,36-070)$ \\
\hline & $\begin{array}{l}\text { 7. para lhe dar informações que ajude a compreender uma } \\
\text { determinada situação }\end{array}$ & $0,33(0,09-0,53)$ \\
\hline & 12. de quem você realmente quer conselho & $0,52(0,31-0,68)$ \\
\hline & 16. para dar sugestões de como lidar com um problema pessoal & $0,54(0,35-0,69)$ \\
\hline \multirow[t]{3}{*}{ Afetiva } & 5. que demonstre amor e afeto por você? & $0,23(-0,01-0,45)$ \\
\hline & 9. que the dê um abraço? & $0,51(0,30-0,70)$ \\
\hline & 19. que você ame e que faça você se sentir querida & $0,70(0,55-0,81)$ \\
\hline \multirow[t]{4}{*}{ Interação social } & 6. para se divertir junto & $0,51(0,31-0,67)$ \\
\hline & 9. com quem relaxar & $0,60(0,42-0,74)$ \\
\hline & 13.com quem distrair a cabeça & $0,53(0,33-0,66)$ \\
\hline & 17. com quem fazer coisas agradáveis & $0,57(0,38-0,71)$ \\
\hline
\end{tabular}

presença de itens redundantes, e abaixo de 0,70 pode refletir baixa consistência interna 4 .

Com a aplicação dos modelos log-lineares, os valores dos índices de confiabilidade deixam de ser uma caixa preta e torna-se possível detalhar a estrutura de concordância com base na distribuição dos pares de respostas para cada pergunta de uma escala, apesar do limitado número de observações. O perfil de distribuição dos pares de respostas revelou particularmente dois padrões: concordância perfeita apenas entre algumas categorias de resposta (semi-independência) e outro de concordância perfeita associado à tendência de respostas altas/baixas na primeira entrevista estarem acompanhadas de respostas altas/baixas na segun- 
Modelos log-lineares selecionados (em negrito) para cada item da escala de apoio social segundo dimensão, 2003.

\begin{tabular}{|c|c|c|c|c|c|c|c|}
\hline \multirow{3}{*}{$\begin{array}{l}\text { Dimensões } \\
\text { Se você precisar, com } \\
\text { que freqüência você } \\
\text { conta com alguém? }\end{array}$} & \multicolumn{7}{|c|}{ Modelos log-lineares } \\
\hline & $\begin{array}{l}\text { Independência } \\
\text { g.l.16 }\end{array}$ & \multirow{2}{*}{$\begin{array}{l}\text { Associação } \\
\text { linear por } \\
\text { linear (ALL) } \\
\text { g.l.15 } \\
\text { G2 }\end{array}$} & \multirow{2}{*}{$\begin{array}{l}\text { Diagonal } \\
\text { g.l.15 } \\
\text { G2 }\end{array}$} & \multirow{2}{*}{$\begin{array}{c}\text { Diagonal+ } \\
\text { ALL } \\
\text { g.l.14 } \\
\text { G2 }\end{array}$} & \multirow{2}{*}{$\begin{array}{c}\text { Triangular } \\
\text { g.l.14 } \\
\text { G2 }\end{array}$} & \multirow{2}{*}{$\begin{array}{l}\text { Semi- } \\
\text { independência } \\
\text { g.l.11 } \\
\text { G2 }\end{array}$} & \multirow{2}{*}{$\begin{array}{l}\text { Semi- } \\
\text { associação } \\
\text { g.l.10 } \\
\text { G2 }\end{array}$} \\
\hline & $\mathrm{G}^{2}$ & & & & & & \\
\hline \multicolumn{8}{|l|}{ Emocional } \\
\hline 2. para a ouvir & 40,6 & 23,7 & 22,5 & 18,5 & 22,4 & 13,5 & 11,9 \\
\hline $\begin{array}{l}\text { 8. para confiar e falar dos } \\
\text { seus problemas }\end{array}$ & 29,9 & 13,8 & 16,0 & 11,4 & 13,5 & 13,2 & 8,9 \\
\hline $\begin{array}{l}\text { 15.compartilhar preocupações } \\
\text { e medos mais íntimos }\end{array}$ & 57,3 & 28,7 & 27,1 & 21,5 & 29,9 & 22,4 & 20,3 \\
\hline 18. que compreenda seus problemas & 64,3 & 30,2 & 14,6 & 12,1 & 14,6 & 4,5 & 4,4 \\
\hline \multicolumn{8}{|l|}{ Material } \\
\hline 1. que a ajude se ficar de cama & 38,1 & 19,9 & 12,0 & 11,3 & 11,6 & 5,0 & 3,1 \\
\hline 4. levá-la ao médico & 44,8 & 27,9 & 17,7 & 16,6 & 16,4 & 12,9 & 12,8 \\
\hline 11. preparar refeições & 56,0 & 17,6 & 32,6 & 19,6 & 31,2 & 22,4 & 13,8 \\
\hline 14. tarefas diárias se ficar doente & 60,3 & 46,6 & 20,5 & 20,5 & 19,5 & 19,9 & 18,4 \\
\hline \multicolumn{8}{|l|}{ Informação } \\
\hline $\begin{array}{l}\text { 3. para lhe dar bom conselho } \\
\text { numa situação de crise }\end{array}$ & 35,3 & 13,7 & 17,9 & 10,6 & 15,8 & 13,8 & 9,2 \\
\hline $\begin{array}{l}\text { 7. para lhe dar informações } \\
\text { que ajude a compreender } \\
\text { uma determinada situação }\end{array}$ & 40,0 & 33,1 & 23,0 & 22,8 & 19,7 & 14,8 & 14,4 \\
\hline $\begin{array}{l}\text { 12. de quem você realmente } \\
\text { quer conselho }\end{array}$ & 53,5 & 36,0 & 20,5 & 12,9 & 20,0 & 9,4 & 8,1 \\
\hline $\begin{array}{l}\text { 16. para dar sugestões de como } \\
\text { lidar com um problema pessoal }\end{array}$ & 39,6 & 18,3 & 21,8 & 15,4 & 15,3 & 14,6 & 10,7 \\
\hline \multicolumn{8}{|l|}{ Afetiva } \\
\hline $\begin{array}{l}\text { 5. que demonstre amor e afeto } \\
\text { por você }\end{array}$ & 28,6 & 23,5 & 19,2 & 18,9 & 18,3 & 3,2 & 2,9 \\
\hline 9. que Ihe dê um abraço & 30,0 & 15,7 & 17,0 & 13,5 & 16,2 & 15,0 & 11,9 \\
\hline $\begin{array}{l}\text { 19. que você ame e que faça } \\
\text { você se sentir querido }\end{array}$ & 45,2 & 13,5 & 17,6 & 9,3 & 17,6 & 6,9 & 3,8 \\
\hline \multicolumn{8}{|l|}{ Interação social } \\
\hline 6. para se divertir junto & 35,4 & 17,1 & 15,8 & 11,1 & 13,8 & 6,5 & 5,5 \\
\hline 10. com quem relaxar & 46,8 & 19,3 & 24,6 & 15,4 & 20,0 & 18,1 & 12,7 \\
\hline 13. com quem distrair a cabeça & 44,6 & 23,8 & 26,5 & 20,1 & 26,3 & 14,0 & 13,2 \\
\hline $\begin{array}{l}\text { 17. com quem fazer coisas } \\
\text { agradáveis }\end{array}$ & 42,9 & 25,1 & 22,7 & 19,2 & 21,3 & 7,8 & 7,8 \\
\hline
\end{tabular}

g.l. = grau de liberdade.

da entrevista (diagonal mais associação linear por linear), com predomínio do primeiro padrão.

O ajuste da estrutura dos dados ao modelo de concordância diagonal é o padrão mais desejável para a validade dos estudos epidemiológicos. Também pode ser considerado bastante satisfatório quando o ajuste se dá pelo modelo de associação linear, pois a discordância nos resultados ocorre entre categorias próxi- mas e caracteriza uma provável variação aleatória nas respostas. Neste estudo, a maioria dos itens (10 itens) envolveu isoladamente ou associados estes dois modelos. Contudo, alguns itens se ajustaram ao modelo de semi-independência, o que faz pensar na possibilidade de introdução de um viés de informação para esses itens, visto que houve maior consistência das respostas para as categorias "às vezes", "quase sempre” e "sempre”. É provável que este 
impacto tenha sido pequeno devido à baixa freqüência das outras categorias de respostas, já que se obteve um alto grau de confiabilidade no escore total.

Diante destes resultados, considerou-se esta escala de apoio social, aplicada sob a forma de entrevista, um instrumento capaz de medir de maneira reproduzível o apoio social nesta população, cumprindo satisfatoriamente esta etapa de avaliação da qualidade das informações sobre o apoio social, principal exposição, no estudo sobre pré-eclâmpsia. Outros estudos devem ser realizados com o objetivo de validar esta escala para mensuração do apoio social numa população de gestantes de baixa renda.

\section{Resumo}

A associação entre apoio social e desfechos relacionados à saúde tem sido objeto de interesse de pesquisadores. $O$ objetivo desse artigo foi avaliar a confiabilidade e estrutura de concordância da escala de apoio social. A confiabilidade teste-reteste foi investigada num grupo de gestantes de maternidade pública $(n=$ 65) por intermédio do coeficiente de correlação intraclasse (CCIC) e kappa ponderado (kw2). Avaliou-se a estrutura de concordância pelos modelos log-lineares. O CCIC da escala foi 0,90 e kw2 variou entre 0,23 e 0,70. Modelos com melhores ajustes foram o de concordância diagonal mais associação linear por linear e de semi-independência. Considerou-se a escala um instrumento capaz de medir, de maneira confiável, o apoio social entre gestantes de baixa renda.

Apoio Social; Estudos de Validação; Modelos Lineares

\section{Colaboradores}

K. S. Silva elaborou o estudo original, supervisionou o trabalho de campo, a análise dos dados e a redação do manuscrito. E. S. F. Coutinho revisou a análise dos dados e a redação do manuscrito.

\section{Agradecimentos}

À Rosane Griep pela contribuição na elaboração deste trabalho. Esta pesquisa recebeu apoio financeiro da Organização Pan-Americana da Saúde (RC/RG-T/ BRA/3192).

\section{Referências}

1. Sherbourne CD, Stewart AL. The MOS social support survey. Soc Sci Med 1991; 38:705-14.

2. Chor D, Griep RH, Lopes CS, Faerstein E. Medidas de rede e apoio social no Estudo Pró-Saúde: pré-testes e estudo piloto. Cad Saúde Pública 2000; 17:887-96.

3. Griep RH, Chor D, Faerstein E, Lopes C. Apoio social: confiabilidade teste-reteste de escala no Estudo Pró-Saúde. Cad Saúde Pública 2003; 19:62534 .

4. Streiner DL, Norman GR. Health measurement scales: a practical guide to their development and use. 2nd Ed. Oxford: Oxford University Press; 1995.

5. Shrout PE, Fleiss JL. Intraclass correlations: use in assessing rater reliability. Psychol Bull 1979; 86:420-8.

6. Fleiss JL. Statistical methods for rates and proportional. New York: John Wiley \& Sons; 1981.

7. May SM. Modeling observer agreement - an alternative to kappa. J Clin Epidemiol 1994; 47:131524 .

Recebido em 28/Jul/2004

Versão final reapresentada em 11/Jan/2005

Aprovado em 15/Fev/2005 